\title{
Successful Outcome of Breast Cancer Patient Refusing Conventional Treatments: A Case Report
}

\author{
Sanghun Lee ${ }^{1}$, Kicheul Sohn ${ }^{2}$, Songha $\mathrm{Chon}^{3}$ \\ ${ }^{1}$ Department of Medical Consilience, Graduate school, Dankook University \\ ${ }^{2}$ Department of Preventive Medicine, Daegu Catholic University Medical Center \\ ${ }^{3}$ Department of Hemato-oncology, Comprehensive and Integrative Medicine Hospital
}

Background: Breast cancer is a treatable disease, but some women reject conventional treatment in favor of unproven "alternative therapies," which may have serious implications for their survival. Therefore, a process is needed to lead them to more appropriate treatment choices.

Case presentation: Here, we present the case of a 51-year-old Korean female diagnosed with early-stage breast cancer (stage IIB, T2N1M0) in Nov. 2015. She refused a standard surgical resection together with chemotherapy and opted instead for moxibustion by nonmedical personnel. Consequently, her preference for alternative therapy without conventional treatment exacerbated her disease. Just a little over a year later, integrative cancer treatment, including chemotherapy based on histological founding, and complementary treatment, comprised of acupuncture, moxibustion, and herbal medicine, were administered for 5 months. Finally, she successfully underwent modified radical mastectomy showing a pathological complete response. She received only adjuvant chemotherapy without any alternative medicine afterwards, and she maintained a good status without recurrence.

Conclusion: In the case of breast cancer patients who are resistant to surgery and chemotherapy, integrative therapy considering adverse effects from conventional treatment should be preferred to bitter opposition to alternative medicine.

Key Words : Breast Cancer, Chemotherapy, Integrative Therapy, Alternative Therapy, Adverse effect

\section{Introduction}

Worldwide, breast cancer is the most common cancer in women and the second most common cancer overall. The National Cancer Institute Surveillance Epidemiology and End Results (SEER) database indicates that the average 5-year survival rate for people with breast cancer is up to $90 \%{ }^{1), 2)}$, which is due to modern treatment modalities comprised of surgery, radiation, and adjuvant drug treatment including chemotherapy, hormonal treatment, and administering trastuzumab, depending on the biologic characteristics of the tumor. Non-invasive (stage 0) and early-stage invasive breast cancers (stages I and II) have a better prognosis than cancers at later stages (III and IV) as follows, with the 5-year relative survival rate decreasing from $99 \%$ in patients with breast cancer located only in the breast to $85 \%$ when spread to the regional lymph nodes, and then $26 \%$ once metastasized to distant parts of the body ${ }^{2)}$. Therefore, early diagnosis and timely

\footnotetext{
- Received : 2 July 2018 - Revised : 8 August 2018 Accepted : 8 August 2018

- Correspondence to : Songha Chon

Department of Hemato-oncology, Comprehensive and Integrative Medicine Hospital, 77, Duryugongwon-ro, Nam-gu, Daegu, Republic of Korea

Tel : +82-53-670-5737, Fax : +82-53-670-6001, E-mail : chonsongha@daum.net
} 
treatment can improve the survival and the quality of life for cancer patients.

Despite outstanding outcomes for conventional treatments, some patients with breast cancer may decline standard treatments due to multiple reasons, probably based on previous experiences and personal values ${ }^{3)}$. Previous studies have shown that patients suffer many psychological conflicts, such as fear of death or disease progression, after being diagnosed with cancer and are worried about the change in the shape of their body after a mastectomy, the adverse effects of chemotherapy, and the deterioration in their quality of life ${ }^{4), 5)}$. These physical and psychological challenges may be associated with various other factors, including social class, education, marital status, living in a rural community, age group, medical co-morbidity, fear of surgery, fear of anesthesia and fear of treatment-related side effects ${ }^{6), 7)}$.

For these reasons, many women with breast cancer tend to prefer a combination of conventional cancer treatment and alternative medicine ${ }^{8)}$, which has been proven to strengthen the immune system and mitigate the side effects of treatment ${ }^{9)}$. However, there are patients with breast cancer who refuse some or all recommended conventional treatments, opting for alternative regimens alone and resulting in significantly worse survival outcomes compared to those receiving standard treatments ${ }^{(6), 10), 11)}$. Among alternative medicines, there are unproven cancer treatments administered by nonmedical personnel that require serious caution ${ }^{10)}$.

The number of patients that decline conventional cancer treatment is substantial enough to need to find solutions. The following report presents a successful outcome in a breast cancer patient resistant to conventional treatment by suggesting integrative therapy considering the adverse effects from chemotherapy.

\section{Case Report}

A 51-year-old Korean female patient presenting a lump in her right breast was diagnosed with early-stage breast cancer (stage IIB, T2N1M0) consisting of a high-grade infiltrating ductal carcinoma with negative estrogen and progesterone receptors and positive HER 2 (3+) in November 2015. A surgical resection was recommended, but she refused it because she was worried of the adverse effects of conventional treatment. Instead, she chose alternative medicine, including moxibustion, conducted by nonmedical personnel. Seven months later, she began neoadjuvant chemotherapy due to her disease progression (Figure 1A). However, she gave up the chemotherapy after 3 cycles of doxorubicin and cyclophosphamide due to toxicity and went back to moxibustion treatment. Five months later, she presented spontaneous discharge from the right painful breast mass, swelling of right arm, and supraclavicular lymph node metastasis (N3 stage; Figure 1B, Figure 2). Consequently, her preference for alternative medicine eventually exacerbated the disease.

The patient visited us in January 2017 to receive integrative cancer therapy to manage her disease. The treatment plan included chemotherapy consisting of weekly paclitaxel with trastuzumab and pertuzumab, based on histological founding, and complementary treatment comprised of acupuncture, moxibustion, and herbal medicine such as Hyangsayangwi-tang to relieve the cancer-related symptoms and reduce the adverse effects of the chemotherapy until May 2017. Her chief complaints were heartburn, abdominal discomfort, pain, fatigue, and hot flushes. A decoction of Hyangsayangwi -tang was prepared from a mixture of chopped crude herbs (Panax ginseng C.A.Meyer (6g), Pinellia ternata (Thunb.) Breitenbach (4g) Amomum cadamomum L (4g), Paeonia lactiflora Pallas (4g), Atractylodes macrocephala Koidzumi (4g), Amomum villosum Lour 


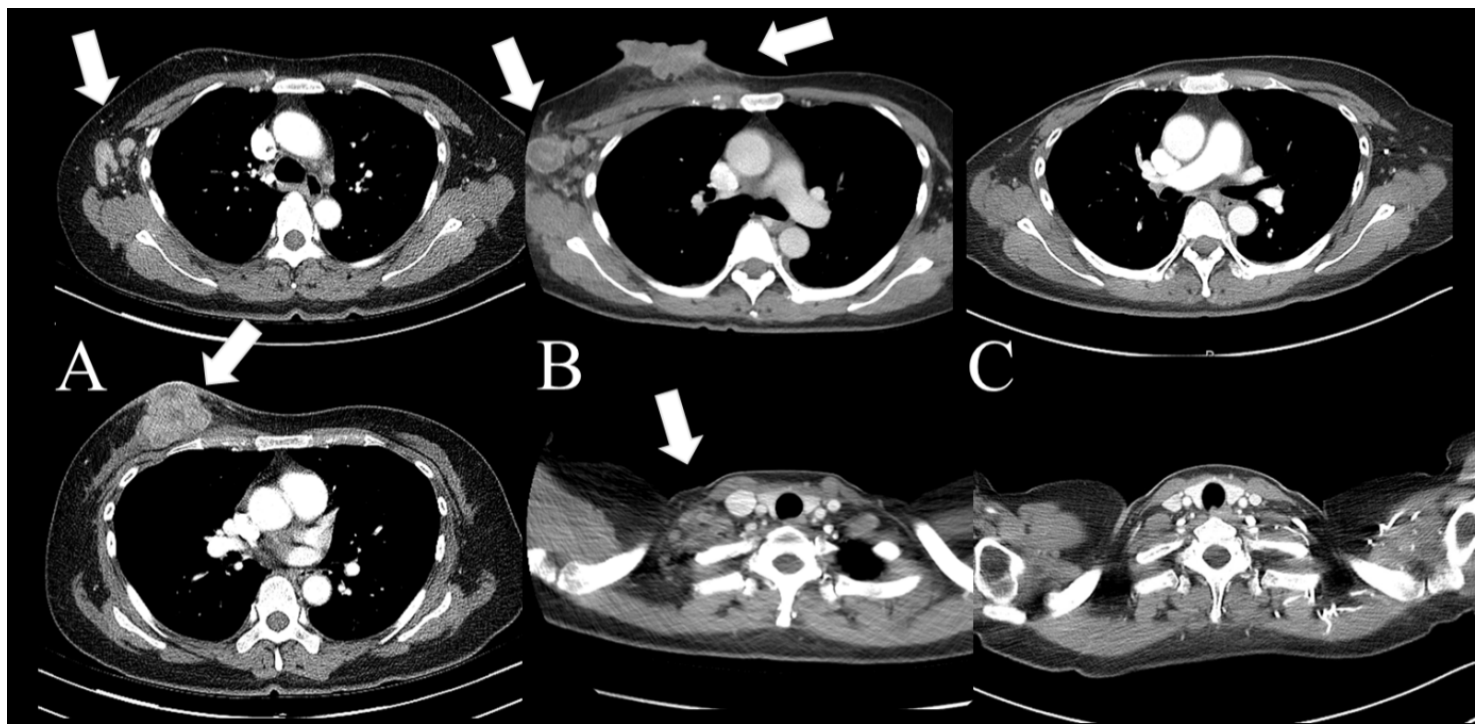

Fig. 1. (A) Chest CT scans dated June 17, 2016 revealed an approximately $50.49 \mathrm{~mm}$ mass in the right breast and enlargement of the axillary lymph nodes. (B) Follow-up CT scans dated January 9, 2017 showed the progression of her breast mass, marked increase of the axillary lymph nodes, and supraclavicular lymph node metastasis after alternative therapy by nonmedical personnel. (C) The recent CT scans dated September 18, 2017 demonstrated no evidence of breast cancer after integrative treatments.

(4g), Crataegus pinnatifida Bunge (4g), Citrus unshiu Markovich (4g), Cyperus rotundus L (4g), Glycyrrhiza uralensis Fischer (4g), Zingiber officinale Roscoe dried (4g), Zizyphus jujuba var. inermis Rehder (3g), Zingiber officinale Roscoe (3g)) that were extracted in water at $100^{\circ} \mathrm{C}$ for 2 hours. The quality of the herbs was tested according to the Korea Food \& Drug Administration and our hospital's standards. Oral administration of $100 \mathrm{ml}$ Hyangsayangwi-tang decoction was prescribed twice a day. She managed to complete neoadjuvant chemotherapy on schedule without dose reduction and adverse effects such as severe fatigue and anorexia which had previously been observed during the first chemotherapy. Paclitaxel-induced neuropathic pain was also managed by acupuncture.

After 6 planned cycles of chemotherapy, she successfully underwent modified radical mastectomy, showing a pathological complete response. She received only adjuvant trastuzumab without any alternative medicine, and no evidence of disease was confirmed by recent CT scans in September 2017 (Figure 1C).

\section{Discussion}

In general, those who prefer alternative cancer therapies tend to have certain characteristics including a younger age, suffering from breast cancer, having higher education and income, living in the Pacific region, and experiencing a progression to a more advanced stage $^{7), 10)}$. Asian breast cancer patients should therefore receive more attention. A Korean study regarding the process to pursue complementary, alternative medicine in patients with breast cancer suggested that the core category is 'endless management of the mind and body for healing', that is, taking desperate efforts of keeping life which are common in patients with incurable diseases like cancer ${ }^{1), 2)}$. This tendency is more prevalent 


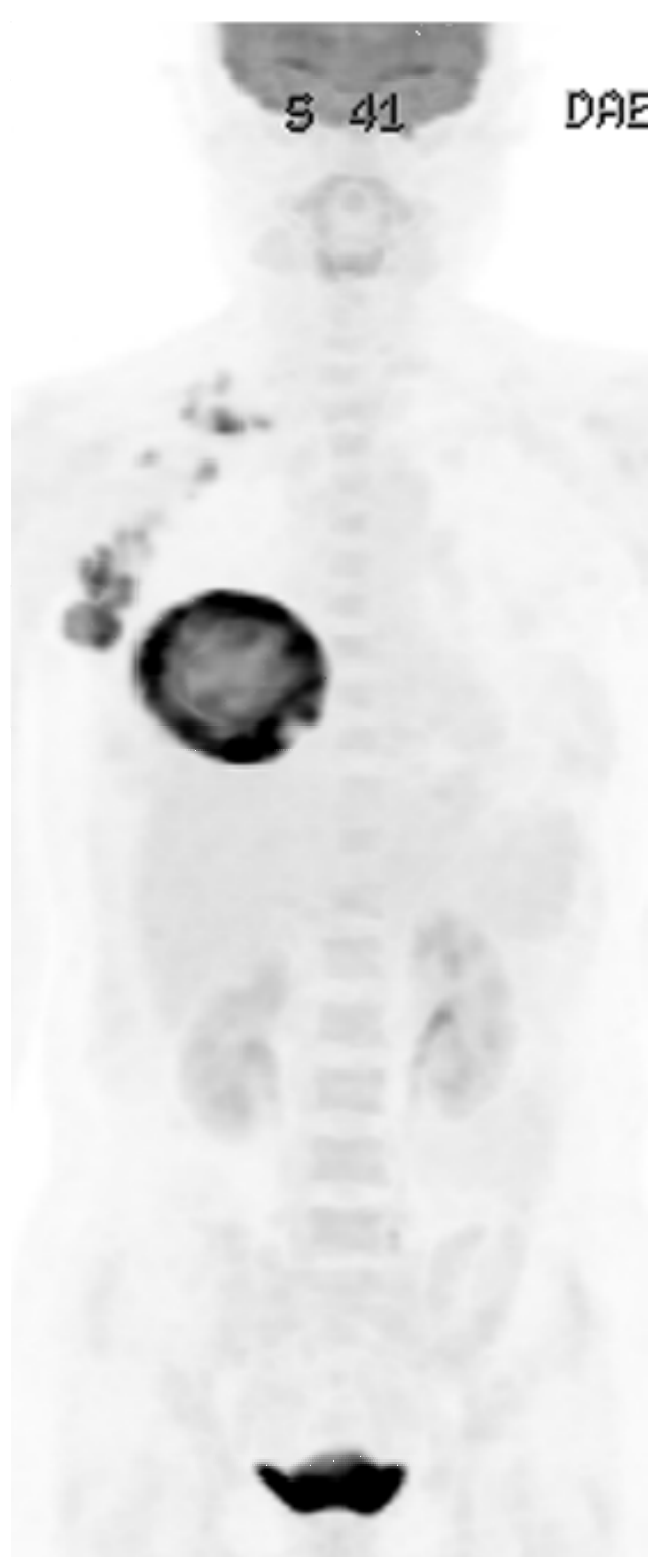

Fig. 2. PET scans dated January 12, 2017 showed hypermetabolic mass in the right breast abutting on the overlying skin with multiple hypermetabolic lymphadenopathies in the right axilla level I-III, subclavicular and supraclavicular area.

in patients with breast cancer than any other type of cancer $^{8)}$. Thus, medical staff will need to understand the beliefs that trigger a decision to decline conventional treatment in favor of alternative therapies, based on a mind to desperately achieve healing. Improved communication between patients and caregivers is needed and is recommended as follows ${ }^{13)}$ : be compassionate and nonjudgmental, give words of hope appropriate to each situation, underscore the patient's decision-making power, give patients time to decide, be supportive but realistic, and so on. However, improvements in these interview methods have much difficulty in ultimately changing patient's decisions. Therefore, an appropriate use of alternative medicine is needed.

Our patient refused conventional treatment twice, even though her disease had progressed. Moreover, moxibustion offered by nonmedical personnel produced burns to her skin, which led to a higher risk of infection. Therefore, evidence-based complementary medicine was properly suggested and performed by doctors of Korean medicine who have been certified by the Korean Ministry of Health and Welfare. She received acupuncture and moxibustion three times a week and was administered herbal medicine, such as Hyangsayangwi-tang (XiangSha-Yang-Wei-Tang in Chinese).

Evidence from various preclinical and clinical studies support the use of herbal medicine or acupuncture in boosting the immune system; relieving pain, fatigue, and cytotoxicity including liver and kidney damage; and inhibiting gastrointestinal toxicity, angiogenesis, and other side effects from chemotherapy in breast cancer $^{14)}$. Acupuncture also improved anxiety, depression, insomnia, neuropathy, and cancer-related hot flushes, which most breast cancer patients suffer from. Hyangsayangwi-tang has not only been traditionally prescribed as a typical decoction to improve the digestive system, but it has also been proven to improve chemotherapy-induced gastrointestinal dysfunction ${ }^{15), 16)}$. In our case, these complementary treatments helped control the cancer-related symptoms and reduce the 
adverse effects of chemotherapy treatment. Therefore, she successfully received planned chemotherapy without dose reduction which finally produced a pathological complete response in spite of locally advanced breast cancer with N3 stage.

In conclusion, patient tailored treatment is important, especially in breast cancer patients refusing conventional treatment in favor of alternative therapy. We hope that this case could encourage individualized integrative therapy for complementing conventional treatment in Korean health care system.

\section{Acknowledgment}

This work was supported by a grant of Comprehensive and Integrative Medicine $R \& D$ project through Comprehensive and Integrative Medicine Institute, funded by the Ministry of Health \& Welfare, Republic of Korea (Grant Number : 090-091-3000-3038-301-320-01)

\section{Disclosure}

The authors report no conflicts of interest in this work.

\section{References}

1. DeSantis CE, Ma J, Goding Sauer A, Newman LA, Jemal A. Breast cancer statistics, 2017, racial disparity in mortality by state. CA: a cancer journal for clinicians. 2017;67(6):439-48.

2. Siegel RL, Miller KD, Jemal A. Cancer Statistics, 2017. CA: a cancer journal for clinicians. 2017;67(1):7-30.

3. van Kleffens $T$, van Leeuwen E, Physicians' evaluations of patients' decisions to refuse oncological treatment. Journal of medical ethics. 2005;31(3):131-6.

4. Bickell NA, Weidmann J, Fei K, Lin JJ, Leventhal
H. Underuse of breast cancer adjuvant treatment: patient knowledge, beliefs, and medical mistrust. Journal of clinical oncology : official journal of the American Society of Clinical Oncology. 2009;27(31):5160-7.

5. Helms RL, O'Hea EL, Corso M. Body image issues in women with breast cancer. Psychology, health \& medicine. 2008;13(3):313-25.

6. Chang EY, Glissmeyer M, Tonnes S, Hudson T, Johnson N. Outcomes of breast cancer in patients who use alternative therapies as primary treatment. American journal of surgery. 2006;192(4):471-3.

7. Chen SJ, Kung PT, Huang KH, Wang YH, Tsai WC. Characteristics of the Delayed or Refusal Therapy in Breast Cancer Patients: A Longitudinal Population-Based Study in Taiwan. PLoS One. 2015;10(6):e0131305.

8. Morris KT, Johnson N, Homer L, Walts D. A comparison of complementary therapy use between breast cancer patients and patients with other primary tumor sites. American journal of surgery. 2000;179(5):407-11.

9. Greenlee H, Balneaves LG, Carlson LE, Cohen M, Deng G, Hershman D, et al. Clinical practice guidelines on the use of integrative therapies as supportive care in patients treated for breast cancer. Journal of the National Cancer Institute. Monographs. 2014;2014(50):346-58.

10. Johnson SB, Park HS, Gross CP, Yu JB. Use of Alternative Medicine for Cancer and Its Impact on Survival. Journal of the National Cancer Institute. 2018;110(1).

11. Joseph K, Vrouwe S, Kamruzzaman A, Balbaid A, Fenton D, Berendt R, et al. Outcome analysis of breast cancer patients who declined evidencebased treatment. World journal of surgical oncology. 2012;10:118.

12. Tae Y, Kwon S, Lee Y, Bae J. Endless Management of Mind and Body for Healing : The Process of 
Journal of Korean Medicine 2018;39(4)

Pursuing Complementary and Alternative Medicine in Patients with Breast Cancer. Korean Journal of Adult Nursing. 2015;27(6):728-41.

13. Citrin DL, Bloom DL, Grutsch JF, Mortensen SJ, Lis CG. Beliefs and perceptions of women with newly diagnosed breast cancer who refused conventional treatment in favor of alternative therapies. The oncologist. 2012;17(5):607-12.

14. Liao GS, Apaya MK, Shyur LF. Herbal medicine and acupuncture for breast cancer palliative care and adjuvant therapy. Evidence-based complementary and alternative medicine : eCAM. 2013;2013:437948.

15. Seo E, Kim S, Bae N, Choi A. Beneficial Effect of Hyangsayangwi-tang on the Cisplatin-Induced Gastrointestinal Dysfunctions in Rats. J Sasang Constitut Med. 2013;25(4):343-58.

16. Song H, Kim D, Kim S. Effect of Hyangsayangwetang and Kamihyangsayangwetang on Antitumor Activity and Reduction of Side Effect. The Journal of Kyung Hee University Medical Center. 1995;11(3-4):320-38.

\section{ORCID}

Sanghun Lee: https://orcid.org/0000-0002-0573-9555 